\title{
Base Stacking Configuration is a Major Determinant of Excited State Dynamics in A.T DNA and LNA
}

\author{
Stanislav O. Konorov ${ }^{1,2}$, H. Georg Schulze ${ }^{1}$, Christopher J. Addison ${ }^{1,2}$, Charles A. Haynes ${ }^{1,3}$, \\ Michael W. Blades ${ }^{*}, 2$ and Robin F.B. Turner ${ }^{*}, 1,2,4$ \\ ${ }^{1}$ Michael Smith Laboratories, The University of British Columbia, 2185 East Mall, Vancouver, BC, V6T 1Z4, Canada \\ ${ }^{2}$ Department of Chemistry, The University of British Columbia, 2036 Main Mall, Vancouver, BC, V6T 1Z1, Canada \\ ${ }^{3}$ Department of Chemical and Biological Engineering, The University of British Columbia, 2360 East Mall, Vancouver, \\ $B C$, V6T 1Z3, Canada \\ ${ }^{4}$ Department of Electrical and Computer Engineering, The University of British Columbia, 2332 Main Mall, Vancouver, \\ BC, V6T 1Z4, Canada
}

\begin{abstract}
Base stacking plays an important role in excited state dynamics in polynucleotides. However, it is poorly understood how stacking geometries influence the formation of and relaxation from excites states. Natural poly $(\mathrm{dA}) \cdot \operatorname{poly}(\mathrm{dT})$ adopts a B-form structure with extensive geometrical overlap between adjacent stacked adenines while the synthetic, locked ribose analogue (LNA), adopts the A-form structure where such overlap between adjacent adenines is reduced. We have used pump-probe transient absorption measurements on DNA and LNA, with excitation at $260 \mathrm{~nm}$ and absorption monitored at 440 and $260 \mathrm{~nm}$, to examine the differences in excited state dynamics in B- and A-form conformations. We observed slow decay times, both early and late stage, from the excited states of B-form and fast decay times from the excited states of analogous homopolymeric A-form structures. Within similar conformations, relaxation times are dependent on the number of stacked adenines as determined by either chain length or sequence. An increase in excited state lifetimes with increase in the number of stacked adenines shows that these excited states can be delocalized over several bases. Thus excited state lifetimes are highly dependent on how the bases are stacked. We conclude from our results that, for identical sequences, conformations that exhibit a high degree of adenine base overlap favor initial cooperative excitation as well as subsequent evolution to delocalized excited states, but hinder the formation of out-of-plane geometries required for fast relaxation to the electronic ground state thus prolonging excited state lifetimes.
\end{abstract}

Keywords: Locked nucleic acids, excited state absorption, DNA conformation, Frenkel exciton, charge-transfer exciton, UV damage.

\section{INTRODUCTION}

A broad absorption maximum is observed around $260 \mathrm{~nm}$ in DNA [1] due to the absorption characteristics of its constituent bases [2]. Molecular orbital calculations [2] and the complexity of circular dichroism (CD) spectra [2-4] suggest that the $260 \mathrm{~nm}$ absorption peak is due to several distinct electronic transitions. For example, Rich and Kasha [1] pointed out that this band involves several $\pi \pi^{*}$ transitions originating from the purine and pyrimidine bases and, within purines, differently polarized $\pi \pi^{*}$ transitions are evident. UV irradiation in this wavelength region therefore creates various excited electronic states in DNA that can relax along different pathways [5-9] some of which may give rise to photoadducts. In addition to the fundamental biophysical interest in DNA photoproduct formation, interest in the excited state dynamics is growing due to the potential for such excited states to affect a number of DNA-related physiological processes, such as mutagenesis. Indeed, UV exposure is known to produce a

*Address correspondence to these authors at the Department of Chemistry, The University of British Columbia, 2036 Main Mall, Vancouver, BC, V6T 1Z1, Canada; E-mails: turner@msl.ubc.ca, blades@chem.ubc.ca variety of deleterious effects on DNA [10], hence an understanding of the formation and relaxation dynamics of excited states in DNA and their dependence on environmental conditions is a subject of great interest [11-14].

Bases in the DNA molecule interact with one another along two roughly orthogonal dimensions: in the plane of the bases by way of base pairing and perpendicular to the base plane by way of base stacking. Base-pairing is affected by the hydrogen bonds that form between complementary bases and forms the basis of the hybridization process $[15,16]$. Basestacking interactions involve several mechanisms, the major ones being dispersion attraction, short-range exchange repulsion, electrostatic interaction, and $\pi \pi$ interactions $[17,18]$. Dispersion forces are generally considered to be the dominant mechanism $[18,19]$, particularly in relation to adjacent adenines due to their relatively small dipole moments. Dispersion forces are related to charge separation by an inverse power law and hence depend strongly on the type of base stacking involved - i.e., the geometrical overlap between stacked bases is important. Geometrical overlap (Fig. 1) is specified in terms of the normal projection of a base onto one of its neighbors (related to shift, slide, and twist) and its proximity to that base (related to roll, tilt, and rise). 


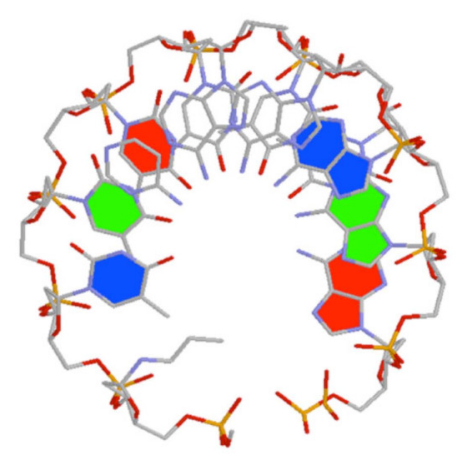

A - Form

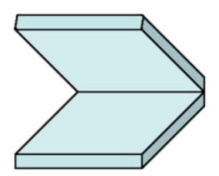

Roll

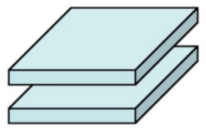

Rise

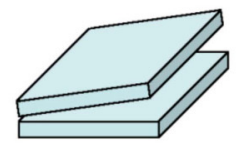

Tilt

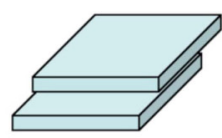

Shift

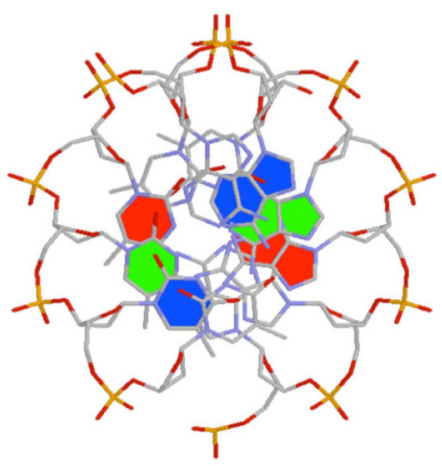

B - Form

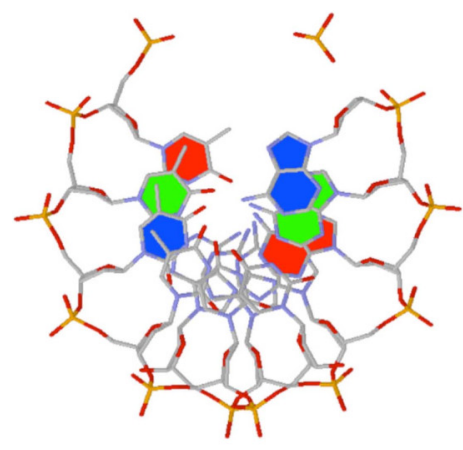

$B^{*}-$ Form

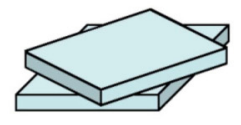

Twist

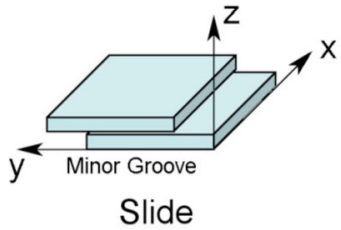

\begin{tabular}{lccc}
\hline Parameters & A-Form & B-Form & B $^{*}$-Form \\
\hline Roll $\left(^{\circ}\right)$ & 12.43 & 1.71 & 4.84 \\
Tilt $\left(^{\circ}\right)$ & 0.02 & 0.0 & 0.19 \\
Twist $\left(^{\circ}\right)$ & 30.30 & 35.96 & 31.24 \\
Rise $(\AA)$ & 3.30 & 3.36 & 3.33 \\
Shift $(\AA)$ & 0.0 & 0.0 & -0.04 \\
Slide $(\AA)$ & -1.39 & 0.45 & -0.51 \\
\hline
\end{tabular}

Fig. (1). Axial views of A, B and B* secondary structures with corresponding geometrical parameters (and their definitions). In each of the axial views, three consecutive bases are colored to emphasize relative orientations and overlap regions; the blue-filled base is at the $3^{\prime}$ end of the colored sequence. The colored bases on either strand are not paired with the colored bases on the opposite strand. The parameter values for the depicted secondary structures are for models included in 3DNA [21].

In single-stranded DNA (ssDNA), the forces involved in base-stacking may be the primary determinants of secondary structure [20], whereas in double-stranded DNA (dsDNA), these forces interplay with the other intermolecular forces, primarily hydrogen bonding, acting through base-pairing of the duplex to determine secondary structure. More specifically, base sequence affects hydrogen bonding and dispersion forces and so influences local molecular conformation. Considering the relationship between conformation and base stacking, for example, A-DNA has a helical diameter a few angstroms larger than that of B-DNA and the bases are arranged closer to the perimeter of the helix rather than closer to the center axis of the helix as in B-DNA [15, 21], leading to different degrees of overlap between bases in these two conformations (Fig. 1). As a consequence, excitation and relaxation dynamics in nucleic acid polymers may be strongly dependent on both base pairing and base stacking.

The extent to which base pairing affects the likelihood and nature of the initial excitation as well as its subsequent relaxation pathways are still unsettled; this is also true for the extent to which base stacking affects excitation and relaxation $[20,22]$. Base pairing causes a blue shift in ${ }^{1} n \pi^{*}$ transitions of the constituent bases and $\mathrm{H}$-bonds deviate from linearity in ${ }^{1} n \pi^{*}$ states, but generally, base pairing does not have a significant effect on ${ }^{1} \pi \pi^{*}$ transition energies and H-bonds remain near linear in the latter states [23]. However, there is evidence, based on vibrational frequency analysis of computationally optimized reference geometries, that the ${ }^{1} \pi \pi^{*}$ state is non-planar in guanine near its Watson-Crick (WC) hydro- gen bonding sites (C6N1C2N3 part) [24]. If the latter, more recent, interpretation is confirmed, the assessment of $\mathrm{H}$-bond planarity in guanine-cytosine (GC) pairs requires reassessment.

UV excitation in a base pair is restricted to a single base [23-25]. The results of gas-phase fluorescence up-conversion spectroscopy show unambiguously that $\mathrm{H}$-bonding reduces the excited state lifetimes of locally excited bases by more than two orders of magnitude to subpicosecond levels [26]. After local ${ }^{1} \pi \pi^{*}$ excitation a ${ }^{1} \pi \pi^{*}$ guanine-to-cytosine charge transfer exciton (CT) develops and electron transfer to the cytosine is followed by single proton transfer. Close to the single proton transfer excited state minimum, a conical intersection (CI) with the ground state occurs that permits relaxation to the electronic ground state [27, 28]. However, femtosecond pump-probe ionization spectroscopy has not provided evidence for such intermolecular electron and/or proton transfers in adenine-thymine (AT) base pairs [8]. Although, based on experimental results [11] and the preceding discussion, base-pairing in $\mathrm{A} \cdot \mathrm{T}$ oligomers is not expected to have significant effects on excited state formation and relaxation, hydrogen bonding with the solvent is known to affect vibrational cooling $[29,30]$ and differential hydration in A-form and B-form nucleic acids [15, 31,32] may play different roles in relaxation processes from excited states in these conformations.

Regarding stacking, it greatly facilitates the transfer and sharing of excited state energies in adenines. The highest oc- 
cupied (HOMO) and lowest unoccupied molecular orbitals (LUMO) normally occur on purines and pyrimidines, respectively, in stacked pairs [33]. Where purines are stacked on purines, and pyrimidines on pyrimidines, the HOMO and LUMO are always on opposite strands [33]. The exception is in sequences of base pairs where adenines are stacked on adenines, then they occur on the same strand [33], making intrastrand $\mathrm{CT}$ excitons possible. Studies using 2-aminopurine, a fluorescent relative of adenine (6-aminopurine), as an energy trap have shown the most efficient excitation energy transfer to occur via neighboring adenines in the stack $[14,34]$. Computational analyses $[20,22,35,36]$, the increase in excited state absorption (ESA) intensities at visible frequencies (435 $\mathrm{nm}$ ) with adenine stack length [37], and different time constants at different emission wavelengths measured in fluorescence decay experiments in $(\mathrm{dA})_{20}$ [38], all indicate that excited state delocalization can occur over several adenines on the same strand. Absorption spectra show that UV absorption in poly $(\mathrm{dA}) \cdot \operatorname{poly}(\mathrm{dT})$ and $(\mathrm{dA})_{20} \cdot(\mathrm{dT})_{20}$ is blue shifted compared to $(\mathrm{dAdT})_{10} \cdot(\mathrm{dTdA})_{10}$ and compared to an equivalent mixture of monomers $[35,39]$. The blue shift, a footprint of coupling between bases [39], results from Franck-Condon excited states being delocalized over adjacent bases as Frenkel excitons $[20,35,36,39,40]$. Because such dipolar coupling depends on the orientation of the transition dipoles, it is sensitive to the geometrical arrangement of the chromophores [41] and, consequently, to the base sequence $[20,39]$. Furthermore, in adenine stacks, because of the small dipole moment compared to other bases and the consequent increased relevance of London dispersion interactions [18], induced dipole-transition dipole interactions also may be important. Clearly, both from theoretical and experimental perspectives, stacking have considerable influence on the formation of excited states.

Stacking also has the potential to affect energy dissipation from these excited states. Out-of-plane geometries are generally considered to be important in allowing nonradiative relaxation to the electronic ground state $[6,23,24$, 30]. Relaxation occurs either via a barrierless CI between a puckered ${ }^{1} \pi \pi^{*}$ state and the electronic ground state $[6,9]$ or via doorway states such as the ${ }^{1} \pi \sigma^{*}$ state along the N9-H reaction coordinate $[7,42]$, requiring an out-of-plane coupling mode [42], or the ${ }^{1} n \pi *$ state involving out-of-plane displacement [43]. Stacking may inhibit the formation of required non-planar geometries thus impeding non-radiative relaxation $[36,44]$. Specifically, since the distance between bases is determined by the trade-off between dispersion attraction and short-range repulsion forces, and because vertical compression of bases is energetically costly [18], stacking may hamper differentially the formation of out-of-plane geometries if bases are stacked differently as they are in Band A-form structures. This is because out-of-plane pucker may occur more easily in adenines when stacked in A-form where there is less overlap between bases compared to Bform where they overlap, and hence are constrained, more.

Of key interest therefore is to better understand how different base stacking geometries govern excited state formation, evolution, and relaxation. Base stacking affects differently electronic structures present in different polynucleotide conformations [45]. The different electronic structures in different conformations influence excited state generation and evolution and hence imply that different base stacking configurations could be evidenced by relative differences in
ESA intensities and lifetimes. Different base stacking configurations may also affect available energy dissipation pathways thus further affecting the intensities and lifetimes of excited states.

Long-lived (ps) excited states have been reported for some sequences of double-stranded and single-stranded DNA containing adenine stacks $[11,13,20,36,37,44]$, but not for stacks of thymines or stacks of mixed sequence [11]. Since the former are in $\mathrm{B}^{*}$-form and the latter in random coil or in B-form, the experimental evidence does suggest the importance of the geometrical arrangement of DNA chromophores in its responses to UV irradiation. However, it is difficult to disentangle sequence effects from conformation effects since base sequence is generally a determinant of conformation. For example it is well-known that poly(dA) poly(dT) and poly(dAdT) poly(dTdA) DNA duplexes, differing only in sequence, have different conformations [46-48]. Therefore, although the effects of primary structure on excited state dynamics have received some attention in the literature $[11,14,37]$, very little data that directly reveal secondary structural effects exist [49]. Specifically, data on single vs double stranded sequences, mixed sequences of $\mathrm{A}$ and $\mathrm{T}$, and strands of varying length are lacking. Furthermore the extant data on single stranded poly(A) and poly $(\mathrm{dA})$ show only a difference in ESA intensity proportional to the percentage of bases stacked in each polymer [49]. No differential effect on lifetimes is observed that can be attributed to differences in the geometrical arrangement of the chromophores, A-type for poly(A) and B-type for poly(dA) [49]. The joint consideration of existing theoretical and experimental work, as outlined above, suggests that one could expect otherwise.

To investigate the influence of base stacking conformation on excited-state relaxation processes in DNA, we have used transient absorption techniques to compare the excited state dynamics of DNA oligomers with and without one or more locked nucleic acid (LNA) monomers. LNA is a modified ribonucleic acid containing a methylene bridge between the $\mathrm{O}^{\prime}$ ' and $\mathrm{C} 4$ ' of the ribose ring, locking the sugar into a $\mathrm{C} 3$ '-endo conformation $[50,51]$. In oligomers containing one or more LNA monomers, the C3'-endo conformation of the locked base influences the preferred sugar conformation of neighboring bases and thereby alters the secondary structure of the entire strand. For example, dsDNA with a $(\mathrm{dA})_{\mathrm{n}} \cdot(\mathrm{dT})_{\mathrm{n}}$ primary structure adopts a B-like secondary structure $\left(\alpha \mathrm{B}^{\prime}-\right.$ or $\mathrm{B}^{*}$-form, with a transition between them occurring around $30{ }^{\circ} \mathrm{C}$ for $300 \mathrm{bp}$ ) with the sugar preferentially in the $\mathrm{C} 2$ 'endo conformation $[46,48,52]$, whereas the LNA-DNA hybrid with the same primary structure has been shown to adopt an A-like secondary structure [51]. LNA therefore offers a considerable advantage in spectroscopic investigations of biophysical phenomena dependent on secondary structure by allowing a means of examining different secondary structural configurations of a given base sequence without altering the solution conditions or temperature.

We present here the first report on the excited state dynamics of LNA and the first comparative study of the transient absorption characteristics of DNA and LNA monomers, dimers and trimers, as well as 12-mers (as single strands, fully hybridized duplexes, as well as A and T homo- and heteropolymers). The results conclusively show that elec- 
tronic excited state dynamics in DNA are highly dependent on the geometrical arrangement of DNA bases, especially adenine, and hence that conformation plays an important role in determining the responses of DNA to UV irradiation. In addition to its biophysical significance, this finding has noteworthy biological implications in that it suggests that different parts of the genetic material may exhibit different conformationally-related robustness against deleterious UV irradiation effects.

\section{MATERIALS AND METHODS}

\section{Oligomer Sample Preparation}

All samples used in this study are defined in Table $\mathbf{1 .}$ Samples I, II, 1, 2 (HPLC purified from Exiqon A/S, Denmark) were used to investigate chain length effects; the remaining samples (standard desalting from IDT Coralville, USA) were used to investigate conformational effects in both single- and double-stranded, and both homo- and heteropolymer samples; only adenines were locked in the LNA oligomers. Note that we follow here the convention of denoting any monomer or oligomer containing one or more locked monomers as "LNA" [51].

Table 1. Oligomer Sequences Used (with Locked Adenine Bases Bold-Underlined), and Putative Secondary Structural Assignments Based on our Survey of the Biophysical Literature. The Secondary Structures of all Single-Stranded Oligomers are Annotated with "Like" to Acknowledge that Care Must be Exercised in Classifying Single-Stranded Oligomers in this Way

\begin{tabular}{|c|c|c|}
\hline Sample ID & $2^{\circ}$ Structure & Sequence \\
\hline I & - & $5^{\prime}-\underline{A}-3^{\prime}$ \\
\hline II & A-like & $5^{\prime}-\underline{A} A-3^{\prime}$ \\
\hline III & A-like & $5^{\prime}-\underline{\mathrm{AAAAA}}-3^{\prime}$ \\
\hline IV & A-like & $5^{\prime}-\mathrm{AAAAA}-3^{\prime}$ \\
\hline $\mathrm{V}$ & A-like & $5^{\prime}-\mathrm{AA} \underline{\mathrm{A} A \mathrm{~A}-3^{\prime}}$ \\
\hline VI & A-like & $5^{\prime}-\mathrm{A} \underline{\mathrm{A}} \underline{\mathrm{A}} \underline{\mathrm{A}} \mathrm{A}-3^{\prime}$ \\
\hline VII & A-like & $5^{\prime}-\mathrm{AAA}$ AAA $\underline{A}$ AA $\underline{A A A}-3^{\prime}$ \\
\hline VIII & A-like & $5^{\prime}-\mathrm{TTT}$ АTT ATA AAA-3, \\
\hline 1 & B-like & $5^{\prime}-\mathrm{AA}-3^{\prime}$ \\
\hline 2 & B*-like & $5^{\prime}-\mathrm{AAA}-3^{\prime}$ \\
\hline 3 & $\mathrm{~B}^{*}$-like & $5^{\prime}-\mathrm{AAAAA}-3^{\prime}$ \\
\hline 4 & $\mathrm{~B}^{*}$-like & 5'-AAA AAA AAA AAA-3' \\
\hline 5 & B-like & 5'-TTT АТT АTA AAA-3' \\
\hline$\alpha$ & Random coil & 5'-TTT ТTT ТTT ТTT-3' \\
\hline A & $\mathrm{B}^{*}$ & $\begin{array}{l}5^{\prime}-\mathrm{AAA} \text { AAA AAA AAA-3' } \\
3^{\prime} \text {-TTT TTT TTT TTT-5' }\end{array}$ \\
\hline B & B & $\begin{array}{l}5^{\prime}-\mathrm{TTT} \text { АTT ATA AAA-3' } \\
3^{\prime} \text {-AAA TAA TAT TTT-5' }\end{array}$ \\
\hline $\mathrm{C}$ & A & $\begin{array}{l}5^{\prime}-\mathrm{AAA} \text { AAA } \text { AAA } \text { AAA-3' } \\
3^{\prime} \text {-TTT TTT TTT TTT-5' }\end{array}$ \\
\hline $\mathrm{D}$ & A & $\begin{array}{l}5^{\prime}-\mathrm{TTT} \text { АTT ATA AAA-3' } \\
3^{\prime} \text {-AAA TAA TAT ТTT-5' }\end{array}$ \\
\hline
\end{tabular}

Single-stranded DNA and LNA oligomers were dissolved in $10 \mathrm{mM}$ phosphate buffer ( $\mathrm{pH} \mathrm{6)}$ to a final concentration of $300,150,100,45$ and $25 \mu \mathrm{M}$ for monomers, dimers, trimers, pentamers, and 12-mers, correspondingly as determined by measuring the absorbance at $260 \mathrm{~nm}$ and 80 ${ }^{\circ} \mathrm{C}$ using a Cary $1 \mathrm{E}$ UV-vis spectrophotometer (Varian, Palo Alto, CA) and then divided into $400 \mu \mathrm{L}$ aliquots. Doublestranded samples were prepared by combining 1:1 molar equivalents of complementary single-stranded oligomers in $10 \mathrm{mM}$ phosphate buffer ( $\mathrm{pH}$ 6) to a final concentration of $25 \mu \mathrm{M}$, then divided into $400 \mu \mathrm{L}$ aliquots, and hybridized in a thermal cycler (Cetus 480, Perkin Elmer, Wellesley, MA). Hybridization was performed by heating the oligomers up to $80^{\circ} \mathrm{C}$ and then cooling them down to $20^{\circ} \mathrm{C}$ at a rate of $1{ }^{\circ} \mathrm{C} / \mathrm{min}$.

\section{Femtosecond Pump-Probe Measurements}

The pump-probe transient absorption measurements were carried out using a femtosecond Ti:Sapphire laser setup (MaiTai master oscillator and SpitFire amplifier, SpectraPhysics, Mountain View, CA) with an optical parametric amplifier (Topas, Light Conversion, Vilnius, Lithuania). The laser generated $130 \mathrm{fs}$ pulses with energy $2 \mathrm{~mJ} /$ pulse at a 1 $\mathrm{kHz}$ repetition rate and $780 \mathrm{~nm}$ central wavelength. The pump beam employed the third harmonic of the main laser radiation at $260 \mathrm{~nm}$ with about $1 \mathrm{GW} / \mathrm{cm}^{2}$ intensity at the sample. The probe beam employed either part of the pump radiation (i.e. $260 \mathrm{~nm}$ ) or visible radiation from the optical parametric amplifier (OPA) with a central wavelength of 440 $\mathrm{nm}$. Polarization of the pump and probe beams were oriented at the magic angle. In measurements where $260 \mathrm{~nm}$ radiation was used for both pump and probe, the beams were not collinear and hence separation for probe detection was effected geometrically. In measurements where the $440 \mathrm{~nm}$ probe was used, the probe and pump beams were collinear and the UV radiation was filtered out for probe detection using UVopaque Plexiglas. Every third pulse of the pump radiation was transmitted using a mechanical chopper. The probe radiation energy was attenuated by at least a factor of $10 \mathrm{com}-$ pared to the pump radiation. The probe signal was detected with an amplified silicon photodiode (Det 210, Thorlabs, Newton, NJ) connected to a lock-in amplifier (Model SR830 DSP, Stanford Research Systems, Sunnyvale, CA). The time delay between pump and probe was scanned in $67 \mathrm{fs}$ steps as sample absorbance was measured at $20^{\circ} \mathrm{C}$ in a thermostated quartz cuvette with a $10 \mathrm{~mm}$ optical path length; the sample was continuously stirred within the cuvette using a magnetically actuated stir bar. The time resolution of all measurements was between 200 and $300 \mathrm{fs}$.

\section{Data Analysis}

Data were manipulated and analyzed using Origin (OriginLab, Natick, MA) and MATLAB (The MathWorks, Natick, MA) software running under Windows XP (Microsoft, Redmond, WA). Graphics of secondary structures were generated, in part, with 3DNA software (freeware from Rutgers, Piscataway, NJ) [21].

\section{RESULTS AND DISCUSSION}

Fig. (2) is a schematic diagram showing postulated energy levels and relaxation pathways for water-solvated stacked adenines in a single-stranded B-form configuration. 
We propose Fig. (2) as a 'model' for reference and discussion purposes since all the excited states and their relaxation pathways could be modified by conformation [49, 53], oligomer length [13, 37], hybridization [8, 11, 22], and hydration [7, 36]. We assume here, following Buchvarov et al. [37], that both individual and cooperative excitation is possible in oligomers such that in well stacked regions, e.g. $80 \%$ of bases in poly $(\mathrm{dA})$ at room temperature $[11,54]$, excitation is cooperative and that in poorly stacked and endregions excitations are localized to individual bases. We also assume, broadly following Bouvier et al. [57], that structural fluctuations due to thermal motions may modify stacking from well-stacked to poorly stack and we furthermore assume that these structural fluctuations occur on timescales overlapping some of those of excited state dynamics.

On the one side are energy levels reflecting a "monomerlike" electronic structure that is not substantively distorted by intra- or inter-molecular interactions. Upon absorption of a UV photon $(240 \mathrm{~nm}-270 \mathrm{~nm})$, a transition occurs to the quasicontinuum of ${ }^{1} \pi \pi^{*}$ electronic states $\left({ }^{1} L_{a}\right.$ and $\left.{ }^{1} L_{b}\right)[42$, $44,55]$ where the ${ }^{1} L_{a}$ HOMO-to-LUMO transition is favored compared to the ${ }^{1} L_{b}$ HOMO-to-LUMO +1 transition [36]. Ultrafast (femtosecond) relaxation then occurs either to the electronic ground state or to lower energy cooperative states whence slower relaxation to the electronic ground state occurs (more below). Ultrafast non-radiative relaxation to the ground state involves nonplanar geometries [6, 23, 24, 30]. Relaxation may proceed directly from the ${ }^{1} \pi \pi^{*}$ states through a barrierless CI with the electronic ground state [6, 9], with C2 out-of-plane pucker near the CI [6], or via ${ }^{1} \mathrm{n} \pi^{*}$ and ${ }^{1} \pi \sigma^{*}$ doorway states through which CIs with the electronic ground state are reached $[6-8,42,56]$. The ${ }^{1} n \pi^{*}$-route is the more dominant in adenine while the ${ }^{1} \pi \sigma^{*}$-route becomes dominant in hydrated adenines [7] and adenine dimers $[7,8]$ (and presumably in poorly stacked poly $(\mathrm{dA})$ since in the latter the electron-withdrawing sugar group may lower the ${ }^{1} \pi \sigma^{*}$ state compared to 9-methyladenine) [43]. These pathways lead to vibrationally hot electronic ground states $\left(\mathrm{S}_{0}{ }^{\mathrm{vib}}\right)$ and further relaxation through vibrational cooling to the ground state $[11,29,30]$. An ultrafast transition may also occur from the ${ }^{1} L_{a}$ state to the ${ }^{1} L_{b}$ state and emission from both at $\sim 307 \mathrm{~nm}$ and $\sim 344 \mathrm{~nm}$, respectively [44].

On the other side are energy levels reflecting electronic structures that are distorted by intra- or inter-molecular interactions, predominantly due to stacking. Upon absorption of a UV photon, a cooperative transition $[36,37]$ occurs to a delocalized excited state with higher energies than the ${ }^{1} L_{a}$ and ${ }^{1} L_{b}$ states. The higher energy of this cooperative excited state is inferred from the blue shift in UV absorption spectra of stacked adenines [35, 40] and energy level splitting [55] due to coupled ${ }^{1} L_{a}$ states $\left({ }^{1} L_{a}{ }^{\prime}\right.$ state). Relaxation from this Frenkel exciton can occur to a localized excited state very similar in geometry to the ${ }^{1} L_{a}$ state and relaxation from there occurs as described above [36]. Relaxation can also occur to other excitons with Frenkel character or those with CT character [36]; in addition, intermediate states between these two are posited to exist [40]. We interpret radiative decay from the localized ${ }^{1} L_{a}$-like state to correspond with the $\sim 310 \mathrm{~nm}$ "Alike" emissions, from the Frenkel excitons with emissions at $\sim 350 \mathrm{~nm}$, and from CT excitons with emissions at $\sim 390 \mathrm{~nm}$ as reported by Kwok et al. [44]. The CT and Frenkel excitons represent local minima on the potential energy surface of the molecule [36]; since barrierless pathways to electronic ground state conformations are not available, relaxation from

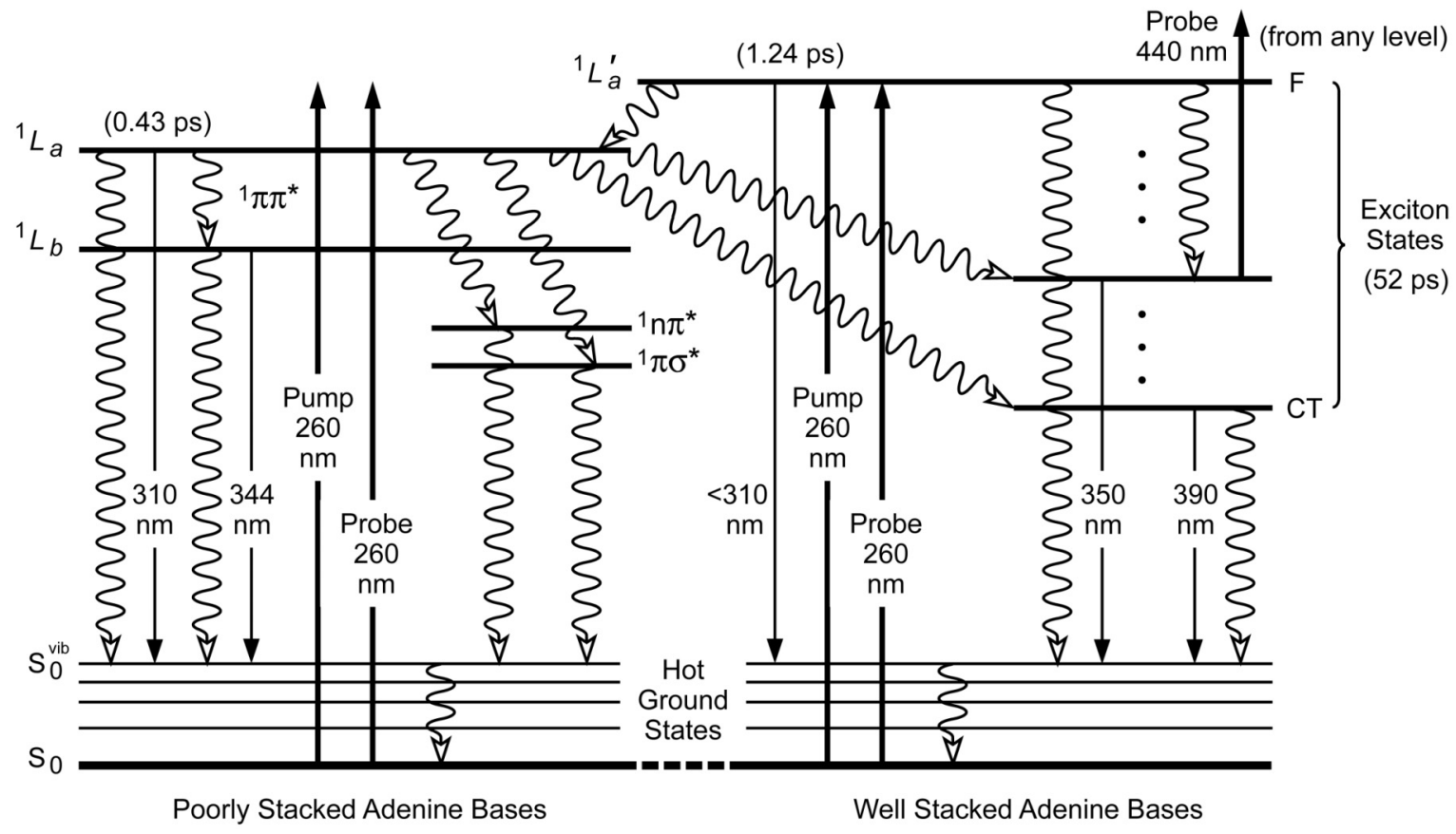

Fig. (2). Energy level diagram showing excited state lifetimes, relaxation pathways, and emission wavelengths inferred and compiled from our transient absorption experiments and literature reports [7, 9, 11, 35-37, 42, 44]. The energy levels on the one side reflect a base under conditions where neighboring adenines are poorly stacked, thus a monomer-like electronic structure prevails; the energy levels on the other side reflect the same base in the same oligomer, but under conditions where neighboring adenines are well stacked and can accommodate delocalized exciton states due to the prevalence of a significantly more distorted electronic structure. 
these states are in the picosecond range $[11,13,36,37,44$, 49].

\section{Excited State Absorption}

The measured relaxation time constants, amplitudes and related statistical parameters for each sample are summarized in Table 2. ESA decay (Fig. 3, Table 2) reveals a single fast relaxation time constant (less then 500 fs) in AMP with a locked ribose unit, which is similar to relaxation data for AMP with a normal ribose reported by others $[11,44]$. This demonstrates that the modified sugar does not affect the relaxation dynamics of the monomer to any significant extent. We note however that differences may exist since the sugar in DNA is an electron-withdrawing group that may lower the ${ }^{1} \pi \sigma^{*}$ state compared to 9-methyladenine [43] and the extra oxygen in LNA may enhance this effect. It is clear, however, that relaxation is ultrafast and that no long-lived excited states are observed.

In contrast, ESA decays (Fig. 3, Table 2) reveal that long-lived excited states are observed when monomers are combined into short oligomers. It is evident from the

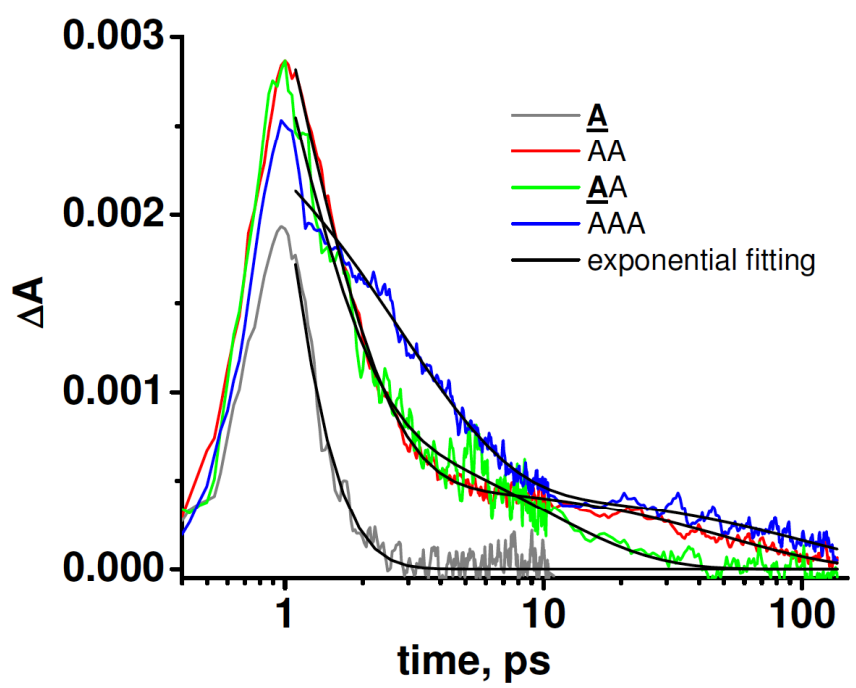

Fig. (3). Transient absorption data (pump $260 \mathrm{~nm}$, probe $440 \mathrm{~nm}$ )

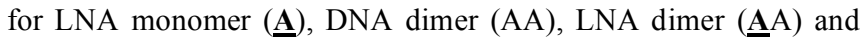
DNA trimer (AAA).

Table 2. Measured Excited State Relaxation and Electronic Ground State Repopulation Time Constants and Amplitudes Pumped at $260 \mathrm{~nm}$ and Probed at $440 \mathrm{~nm}$ or $260 \mathrm{~nm}$, Respectively. The Uncertainties are +/- Boundaries at 95\% Confidence Intervals; the Amplitudes, Denoted $A_{1}$ and $A_{2}$, Refer to the Initial Intensities (in Arbitrary Units) at $t=0$ of the Fitted Exponential Decays Characterized by $\tau_{1}$ and $\tau_{2}$, Respectively

\begin{tabular}{|c|c|c|c|c|c|}
\hline Sample ID & Sample Composition* & $\tau_{1}(\mathrm{ps})$ & $\mathbf{A}_{1}$ (au) & $\tau_{2}(\mathrm{ps})$ & $\mathbf{A}_{2}(\mathbf{a u})$ \\
\hline I & $\mathrm{d} \underline{\mathbf{A}}$ & $0.42 \pm 0.04$ & $22 \pm 2$ & - & - \\
\hline II & $\mathrm{d} \underline{\mathbf{A}} \mathrm{dA}$ & $0.7 \pm 0.1$ & $20 \pm 2$ & $10 \pm 2$ & $8 \pm 1$ \\
\hline III & $\mathrm{d} \underline{\mathbf{A}}_{2} \mathrm{dA}_{3}$ & $1.0 \pm 0.1$ & $86 \pm 4$ & $91 \pm 15$ & $9 \pm 1$ \\
\hline IV & $\mathrm{d} \underline{\mathrm{A}} \mathrm{dAA}_{4}$ & $1.3 \pm 0.1$ & $55 \pm 3$ & - & - \\
\hline $\mathrm{V}$ & $\mathrm{d} \underline{\mathrm{A}} \mathrm{dA}_{4}$ & $1.2 \pm 0.1$ & $73 \pm 5$ & $19 \pm 5$ & $11 \pm 2$ \\
\hline VI & $\mathrm{d} \underline{\mathbf{A}}_{2} \mathrm{dA}_{3}$ & $1.2 \pm 0.1$ & $43 \pm 3$ & - & - \\
\hline VII & $\mathrm{dA}_{9} \mathrm{~d}_{3}$ & $0.73 \pm 0.09$ & $17 \pm 1$ & $8 \pm 3$ & $1.6 \pm 0.3$ \\
\hline VIII & $\mathrm{dA}_{3} \mathrm{~d}_{\mathbf{A}_{3}} \mathrm{dT}_{6}$ & $0.55 \pm 0.07$ & $11 \pm 1$ & $11 \pm 2$ & $4.1 \pm 0.4$ \\
\hline 1 & $\mathrm{dA}_{2}$ & $0.9 \pm 0.1$ & $26 \pm 1$ & $52 \pm 7$ & $4.7 \pm 0.3$ \\
\hline 2 & $\mathrm{dA}_{3}$ & $2.8 \pm 0.3$ & $18 \pm 1$ & $101 \pm 24$ & $4.3 \pm 0.5$ \\
\hline 3 & $\mathrm{dA}_{5}$ & $1.9 \pm 0.1$ & $76 \pm 3$ & $75 \pm 29$ & $22 \pm 8$ \\
\hline 4 & $\mathrm{dA}_{12}$ & $1.24 \pm 0.07$ & $14.8 \pm 0.4$ & $52 \pm 5$ & $4.1 \pm 0.1$ \\
\hline 5 & $\mathrm{dA}_{6} \mathrm{dT}_{6}$ & $0.58 \pm 0.08$ & $12 \pm 1$ & $12 \pm 2$ & $3.9 \pm 0.4$ \\
\hline$\alpha$ & $\mathrm{dT}_{12}$ & $2.02 \pm 0.09$ & $10 \pm 1$ & - & - \\
\hline A & $\left(\mathrm{dA}_{12}\right) \cdot\left(\mathrm{dT}_{12}\right)$ & $1.2 \pm 0.1$ & $14 \pm 1$ & $31 \pm 7$ & $2.9 \pm 0.3$ \\
\hline B & $\left(\mathrm{dA}_{6} \mathrm{dT}_{6}\right) \cdot\left(\mathrm{dT}_{6} \mathrm{dA}_{6}\right)$ & $0.77 \pm 0.1$ & $12 \pm 1$ & $14 \pm 2$ & $4.3 \pm 0.4$ \\
\hline $\mathrm{C}$ & $\left(\mathrm{dA}_{9} \mathrm{~d}_{3}\right) \cdot\left(\mathrm{dT}_{9} \mathrm{dT}_{3}\right)$ & $1.4 \pm 0.2$ & $11 \pm 1$ & $9 \pm 3$ & $3 \pm 1$ \\
\hline $\mathrm{D}$ & $\left(\mathrm{dA}_{3} \mathrm{~d}_{\mathbf{A}_{3}} \mathrm{dT}_{6}\right) \cdot\left(\mathrm{dA}_{6} \mathrm{dT}_{6}\right)$ & $0.7 \pm 0.1$ & $12 \pm 1)$ & $16 \pm 3$ & $4.0 \pm 0.4$ \\
\hline $\mathrm{UV}_{4}$ & $\mathrm{dA}_{12}$ & $1.83 \pm 0.02$ & $-40 \pm 1$ & $71 \pm 1$ & $-8 \pm 1$ \\
\hline $\mathrm{UV}_{\mathrm{A}}$ & $\left(\mathrm{dA}_{12}\right) \cdot\left(\mathrm{dT}_{12}\right)$ & $2.2 \pm 0.1$ & $-60 \pm 7$ & $116 \pm 4$ & $-11 \pm 2$ \\
\hline $\mathrm{UV}_{\mathrm{VII}}$ & $\mathrm{dA}_{9} \mathrm{~d} \underline{\mathbf{A}}_{3}$ & $2.12 \pm 0.03$ & $-45 \pm 1$ & $17.9 \pm 0.3$ & $-13 \pm 2$ \\
\hline $\mathrm{UV}_{\mathrm{C}}$ & $\left(\mathrm{dA}_{9} \mathrm{~d}_{3}\right) \cdot\left(\mathrm{dT}_{9} \mathrm{dT}_{3}\right)$ & $2.22 \pm 0.05$ & $-61 \pm 5$ & $113 \pm 3$ & $10 \pm 1$ \\
\hline
\end{tabular}

*We refer to locked adenylic acid nucleotides as d $\mathbf{A}$ (instead of r $\underline{\mathbf{A}}$, for e.g.) for simplicity here and throughout the text, even though the locked monomer is derived from a ribonucleotide precursor and is not truly a 2'-deoxynucleotide. 
literature $[11,44]$ and our data for the monomer and short oligomers (Table 2), that at least two adjacent adenines are required for biphasic (i.e. picosecond and tens of picoseconds) relaxation dynamics to be observed. Further, if there are more than two adjacent adenines, both fast and slow relaxations $\left(\tau_{1}\right.$ and $\tau_{2}$, respectively, where they exist for a given sample) appear to be affected by the number of adjacent adenines. This observation confirms that excited state dynamics can involve interactions between adjacent bases. Analysis of the data presented in Table 2 also reveals profound differences between A-form and B-form conformations: although biphasic responses can occur in both conformations, both $\tau_{1}$ and $\tau_{2}$ relaxation times are truncated in nearly all A-form conformations studied. The only exception being for Sample III which exhibits a relatively longer $\tau_{2}$. The reason for this is unclear, however this is the only sample studied so far that incorporates two adjacent locked bases, the impact of which (especially in such a short oligomer) on the structure and solvation, and hence the excited state dynamics, may be quite different from that of oligomers without adjacent locked bases. Nevertheless, the dependence of the biphasic de-excitation and the increase in excited state lifetimes on the presence of stacked adenines and on their geometrical overlap discussed above are further supported by ESA in longer oligomers (Fig. 4): long-lived excited states exist in addition to the short-lived ones, and in A-form structures they both are much shorter than in B-form structures. Within conformations, relaxations times are dependent on chain length and sequence. This observation confirms that the excited state dynamics between adjacent bases depend on how they are stacked.

The key observation here is that, for identical sequences, conformation determines the excited state dynamics in nucleic acids. Therefore, our findings provide support for contentions that long-lived excited states are dependent on the appropriate stacking of adenines [44] that ensures sufficient geometric overlap between them [18]. A second important observation is that our data are consistent with some cooperative excitations occurring in adjacent adenines when they are stacked appropriately [37, 44]. This contributes additional experimental data to the discussion as to whether the initial excitation is localized [11,22] or delocalized [35, 36]. It is therefore interesting to consider in more detail which attributes of nucleic acid conformation determine the evolution and decay of excited states and how they may do so. Fig. (1) illustrates the pronounced differences in the geometry of stacking between A- and B-form structures. Therefore one needs to keep in mind the possibility that the excited states in A-form, where there is much less geometric overlap between bases, may be different from those in B-form.

Regarding long-lived excited states, the charge distributions and charge resonances as they pertain to Frenkel and CT excitons also may be considerably affected by the geometrical overlap between bases. For example, calculations involving B-DNA indicate that intermonomer charge transfer occurs between stacked adenines in water [36], but how the transfer occurs and where the charges eventually come to reside on adjacent bases may be very different in LNA. Comparing Samples VII and 4, equivalent except for conformation, it is observed from $\mathrm{A}_{2}$-values in Table 2 that overall the long-lived excited state(s) in $\mathrm{B}^{*}$-form $(4.1 \pm 0.1 \mathrm{x}$ $\left.10^{-4} \mathrm{au}\right)$ is generated more readily than in A-form $(1.6 \pm 0.3$ x $\left.10^{-4} \mathrm{au}\right)$. Since we are not able to identify the particular excited state(s) generated (Fig. 2), we simply infer that delocalized states are generated more easily in adenine stacks with adequate geometric overlap.

In contrast, adequate geometric overlap means that relaxation in $\mathrm{B}^{*}$-form ought to be hindered compared to Aform hence producing the longer $\tau_{2}$ lifetimes in the former structures. Non-planar geometries permit relaxation to the electronic ground state via CIs $[6,23,24,30]$. The $\mathrm{C} 2$ outof-plane pucker, for example, provides access to the electronic ground state from the ${ }^{1} \pi \pi^{*}$ excited state [6]. Adenines stacked in B-form are structurally constrained making these out-of-plane deformations potentially more difficult $[36,44]$ than in LNA (Fig. 1) thus accounting for the observed differences in DNA and LNA delocalized excited state lifetimes. In B-DNA, excitation remains delocalized over several tens of picoseconds despite thermal fluctuations since some interatomic distances contract while others expand resulting in the maintenance of overall coupling [57]. In LNA this may not be the case because thermal fluctuations can more readily produce localized conformations where out-of-plane deformations are not structurally constrained. However, deexcitation may eventually occur in B-DNA, in addition to emission [44], when intraband scattering causes the propagation of a cooperative state to an end-region or poorly stacked region where out-of-plane deformations can occur more easily.

Short relaxation times in A-form and B-form structures are also interesting since they provide evidence for delocalized excitation. Due to electron correlation, excitation is cooperative in regions of well-stacked adenines as inferred from computational studies $[35-37,58]$ and from the blue shift in UV absorption spectra compared to an equivalent sample of monomers [35, 58]; electron correlation should also reduce the absorption cross-section of adenine stacks thereby diminishing absorption intensity. The preceding considerations are consistent with the results shown in Table $\mathbf{2}$ and Fig. (4a, b) for fast $\left(\tau_{1}\right)$ relaxation times in Samples VII and 4. In regions of well-stacked adenines, excitation occurs to the ${ }^{1} L_{a}$ ' delocalized state and in poorly stacked regions to the localized ${ }^{1} L_{a}$ state. Consistent with the explanation given earlier for $\tau_{2}$, ultrafast relaxation to the electronic ground state made accessible via CIs involving nonplanarity is hindered in stacking geometries where non-planarity is more structurally constrained - here B-form as opposed to A-form - therefore the increase in $\tau_{1}(0.73 \pm 0.09$ to $1.24 \pm 0.07 \mathrm{ps})$. The reduced electronic ground state absorption cross-section of adenine stacks means fewer excited states are formed and accounts for the decrease in $\mathrm{A}_{1}\left(17 \pm 1 \times 10^{-4}\right.$ to $14.8 \pm 0.4 \mathrm{x}$ $\left.10^{-4} \mathrm{au}\right)$ from A-form to B-form structures. The evidence indicates that adenines are coupled both before (UV absorption) and after (ESA dependence on adenine chain length) Franck-Condon excitation.

A comparison of Samples 1 and 4 shows similar absorbances $\left(4.7 \times 10^{-4}\right.$ vs $\left.4.1 \times 10^{-4} \mathrm{au}\right)$ and lifetimes $(52 \pm 7 \mathrm{vs}$ $52 \pm 5 \mathrm{ps}$ ) for $\tau_{2}$ indicating that the majority of long-lived excited states formed in single-stranded poly(dA) DNA involve two adenines and may therefore be $\mathrm{CT}$ excitons. Absorbances $\left(26 \times 10^{-4} v s 14.8 \times 10^{-4} \mathrm{au}\right)$ and lifetimes $(0.9 v s$ $1.24 \mathrm{ps})$ for the short-lived $\left(\tau_{1}\right)$ excited states are different indicating that absorption in the dimer is easier and decay 
(a)

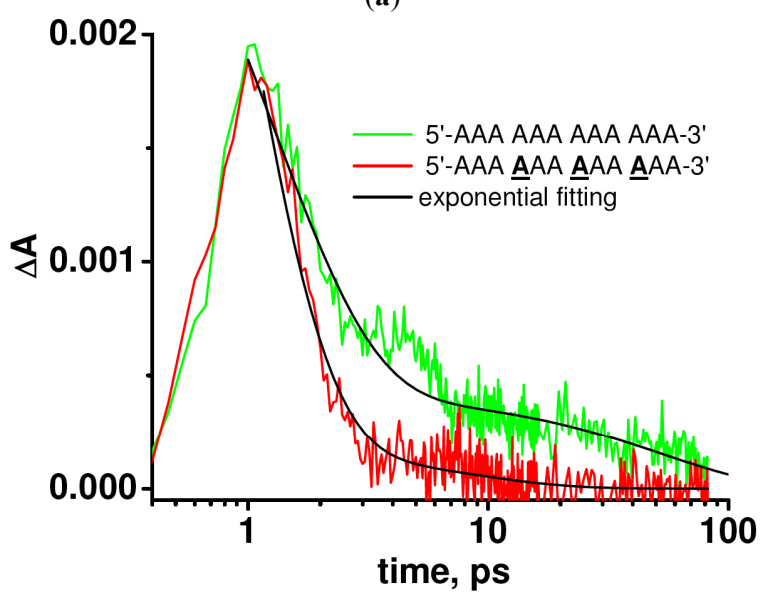

(b)

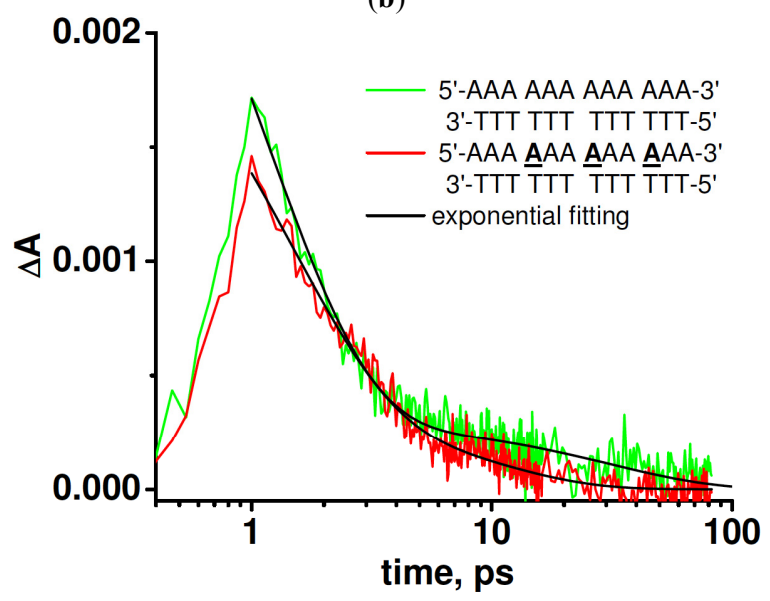

(c)

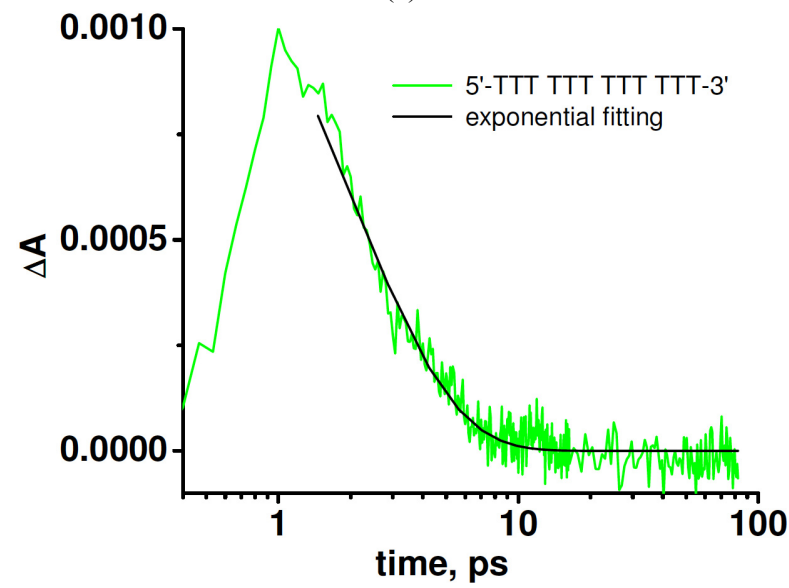

(d)

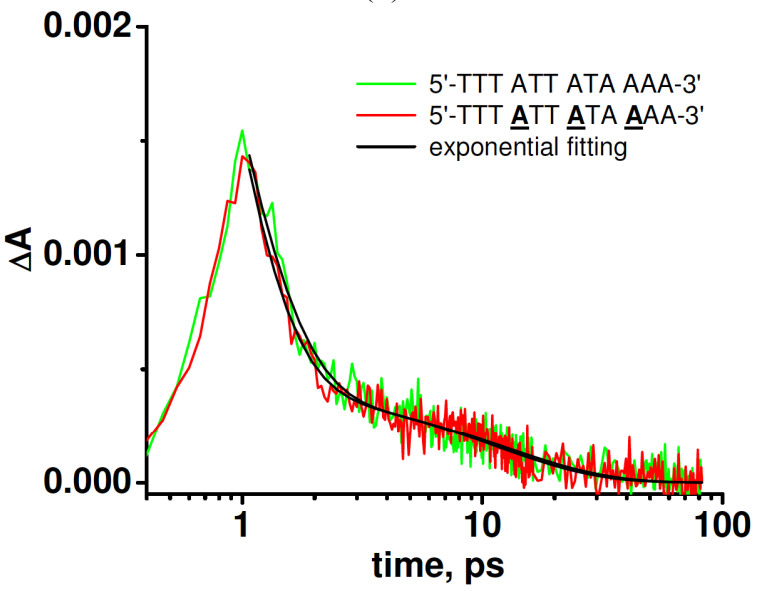

(e)

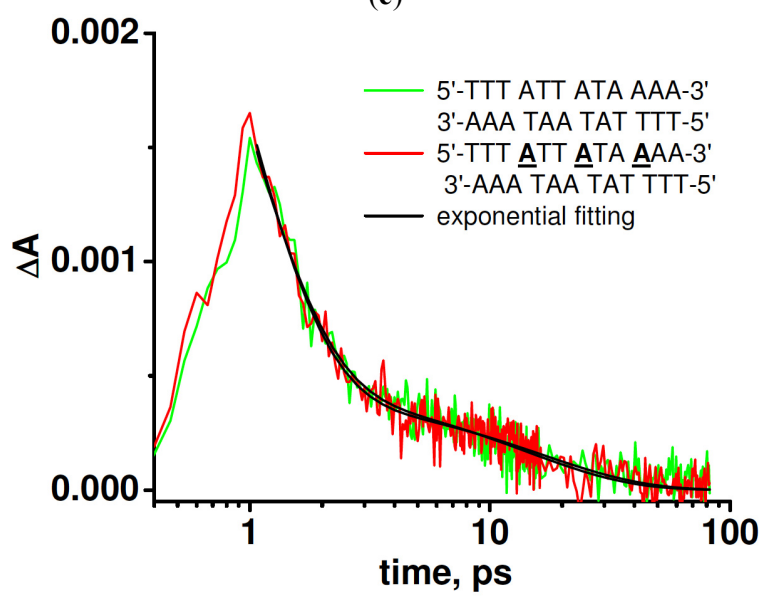

Fig. (4). Transient absorption data (pump $260 \mathrm{~nm}$, probe $440 \mathrm{~nm}$ ) showing single- and double stranded homopolymeric samples (a) poly(dA), (b) poly (dA) poly(dT), (c) poly(dT), and (d) single- and (e) double stranded heteropolymeric samples. The effect of conformation (B*-form, DNA vs A-form, $\underline{\mathbf{L N A}}$ ) on excited state dynamics is very pronounced for stacked adenines. See text for details.

faster because only two bases are involved, while in the dodecamer more than two bases are involved. In other short DNA oligomers (Samples 2 and 3), both time constants are longer than in the less rigid dodecamer (Sample 4). The reduction in lifetimes in longer ssDNA strands therefore probably reflects thermal structural fluctuations within a given conformation which have been shown by Bouvier, et al. [57] to reduce mixing between monomer excited states of the individual bases and hence the spatial extent of the exciton. Bouvier, et al. [57] also showed that both the spatial extent of exciton states and their lifetimes are sensitive to such structural fluctuations, all of which are also dependent on the base sequence. Taken together, short relaxation times $\left(\tau_{1}\right)$ in oligomers of varying length, like those for different conformations discussed above, also provide evidence for cooperative excitation. 
The results (Table 2, Fig. 4a, b) from double-stranded poly(dA)'poly(dT) DNA and LNA (Samples A and C) are qualitatively similar to those observed for single-stranded poly(dA) DNA and LNA (Samples VII and 4) and confirm that, given identical sequences, how bases are stacked has a major influence on their relaxation dynamics. However, both base pairing and the presence of thymine may modify the extent and nature of that influence. Single-stranded poly(dT) DNA (Sample $\alpha$; Fig. 4c), exhibits a single fast relaxation time $\left(\tau_{1}=2.02 \pm 0.09 \mathrm{ps}\right)$ at $440 \mathrm{~nm}$, but repopulation of the ground state reveals a biphasic response attributed to poly(dT) (see below). These results are consistent with previous findings [11].

The mixed purine/pyrimidine sequences (Samples VIII, 5, B, D; Fig. 4d, e) show a decrease relative to the homopolymers (Samples VII, 4, A, C) in transient absorption $\tau_{1}$-components (from range $0.55-0.77$ to range $0.73-1.4$ ps). Their $\tau_{2}$-components (range $11-16 \mathrm{ps}$ ) are intermediate between DNA homopolymers (Samples 4, A; range 31-52 ps) and LNA homopolymers (Samples VII, C; range $8-9$ ps). This complexity is difficult to explain. One possibility is that it reflects a disruption of stacking due to thermal fluctuations in mixed sequences. Persistence length estimates from rotational decay times of transient electric birefringence measurements show that in poly(dT) the helical rise of 0.52 $\mathrm{nm}$ is inconsistent with stacking and that order is mainly due to torsional restrictions within the phosphodiester backbone, not sufficiently rigid in itself to prevent random coil structures, whereas in poly $(\mathrm{dA})$ the much greater intrinsic rigidity is due mainly to purine stacking [59-61]. We would therefore expect thermally induced disorder to be greater in mixed sequences compared to homopolymers because any thymines adjacent to adenines will cause a loss of rigidity in such mixed sequences; this is consistent with theoretical observations that the delocalization length of excitons are more affected by disorder in polymers with alternating base sequences [57]. Given more thermally-induced structural disorder, the $\tau_{1}$-component will be affected relatively more because the Frenkel exciton is delocalized over more than two bases while the $\tau_{2}$-component will be affected relatively less if it is primarily a CT exciton extending over two bases as discussed above. In addition, the larger dipole moment of thymine compared to adenine [18] may constrain the response of dispersion forces to electronic transition moments, in adjacent remnant adenine stacks, hence reducing lifetimes compared to similar length homopolymers.

\section{Ground State Repopulation}

Pumping with $260 \mathrm{~nm}$ light and then probing at reduced intensity with the same wavelength light reveals information about ground state repopulation rates. Ground state repopulation should reflect the effects of all the excited states created in a molecule and their respective energy dissipation pathways. It thus provides, in conjunction with ESA, a unique opportunity for 'double-entry bookkeeping' of excited states within the limits of experimental error. In the case of singleand double-stranded DNA and LNA with poly(dA) strands (Samples $\mathrm{UV}_{4}, \mathrm{UV}_{\mathrm{A}}, \mathrm{UV}_{\mathrm{VII}}, \mathrm{UV}_{\mathrm{C}}$ ), two repopulation timescales are observed as evident in Fig. (5).

The fast repopulation time $\left(\tau_{1}=1.83 \pm 0.02 \mathrm{ps}\right)$ for Sample 1 (Fig. 5a) must reflect all the ultrafast decay processes that occur after the pump pulse, including H-bond mediated solvent-assisted vibrational cooling of the electronic ground state $[11,29,30]$. Ultrafast internal conversion for adenine occurs in about $\sim 0.5$ ps $[11,29,30,44]$ and vibrational cooling of the electronic ground state takes less than 2 ps [11,29, $30]$, values consistent with our findings. The fast repopulation time $\left(\tau_{1}=2.12 \pm 0.03\right.$ ps $)$ in Sample $U_{\text {VII }}$ (Fig. 5b) is $\sim 300$ fs longer than that of Sample $\mathrm{UV}_{4}$. This may indicate that: (i) decay occurs via the same routes as in Sample 1, but one or more of them is/are slower; (ii) a different, and slower, decay pathway is dominant; (iii) a different decay pathway exists that is not accessible in Sample $\mathrm{UV}_{4}$; and (iv) vibrational cooling may be slower in A-form than in B-form structures.

Given the imputed role of H-bonds in solvent-assisted vibrational cooling $[11,29,30]$ and the very different extent and nature of hydration shells in A- and B-form nucleic acid conformations with A-form being more dehydrated [15, 31, 32], also true for LNA [62], one may expect vibrational cooling to be affected differently in these structures. Specifically, molecular dynamics simulations of DNA in water show that the N7 position in adenine is hydrated only about $19 \%$ of the time whereas other H-bonding sites in both adenine and thymine are hydrated $>50 \%$ of the time [63]. Consequently, with LNA being less hydrated than DNA [63], adenine may be more affected in the A-from than thymine leading to longer vibrational cooling times for adenine in LNA, but not for thymine. This interpretation is consistent with the differences in fast repopulation times mentioned above and with the lack of differences observed (Fig. 5c, d) between dsDNA (Sample $\mathrm{UV}_{\mathrm{A}} ; \tau_{1}=2.2 \pm 0.1 \mathrm{ps}$ ) and dsLNA (Sample $\mathrm{UV}_{\mathrm{C}}$; $\tau_{1}=2.22 \pm 0.05 \mathrm{ps}$ ) where the repopulation time is dominated by the longer cooling times of thymine $[29,30]$ (Sample $\alpha ; \tau_{1}=2.02 \pm 0.09 \mathrm{ps}$ ).

Likewise, the slow repopulation times $\left(\tau_{2}=116 \pm 4 \mathrm{ps}\right.$, $\tau_{2}=113 \pm 3$ ps) for Samples $U_{V_{A}}$ and $U_{V_{C}}$ (Fig. $5 \mathbf{c}, \mathbf{d}$ ), respectively, also reflect dominant thymine repopulation of the electronic ground state $\left(\tau_{2}=103 \pm 18 \mathrm{ps}\right.$ [11]) occurring via an unidentified singlet intermediate [11] thus not seen in ESA from Sample $\alpha$. The repopulation times $\left(\tau_{2}\right)$ for Samples $\mathrm{UV}_{4}(\sim 71 \mathrm{ps})$ and $\mathrm{UV}_{\mathrm{VII}}(\sim 18 \mathrm{ps})$ are longer than the excited state decay times $\left(\tau_{2}\right)$ for Samples $4(\sim 52 \mathrm{ps})$ and VII $(\sim 8 \mathrm{ps})$, suggesting the likelihood that unidentified intermediates not probed at $440 \mathrm{~nm}$ also exist in these samples.

Although base stacking occurs in both A-form and Bform DNA, the type of stacking is qualitatively quite different in these two conformations. As shown in Fig. (1), B*form exhibits much greater overlap between adjacent bases than A-form. Delocalized excited state formation, that is, both excimer formation [64] (CT exciton) and Frenkel exciton formation $[20,57]$ between adjacent bases are dependent on their relative geometry (i.e. positions and orientations). Therefore exciton formation and decay are expected to depend on conformation given identical bases sequences. We infer from ESA intensities that delocalized excitations are less likely to occur in LNA $\left(\mathrm{A}_{1}+\mathrm{A}_{2}=18.6 \times 10^{4} \mathrm{au}\right)$ compared to DNA $\left(\mathrm{A}_{1}+\mathrm{A}_{2}=18.9 \times 10^{-4} \mathrm{au}\right)$ relative to the total number of excitations in LNA $\left(\mathrm{A}_{1}+\mathrm{A}_{2}=58 \times 10^{-4} \mathrm{au}\right) \mathrm{com}-$ pared to DNA $\left(\mathrm{A}_{1}+\mathrm{A}_{2}=48 \times 10^{-4}\right.$ au) as inferred from ground state repopulation (i.e. $\sim 33 \% v s \sim 40 \%$, respectively). Thus, in addition to relaxation to the ground state from ex- 
(a)

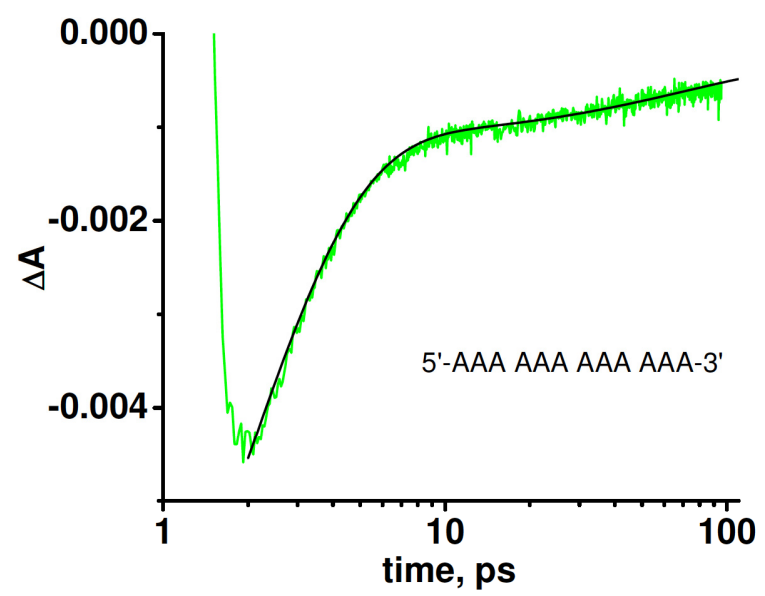

(c)

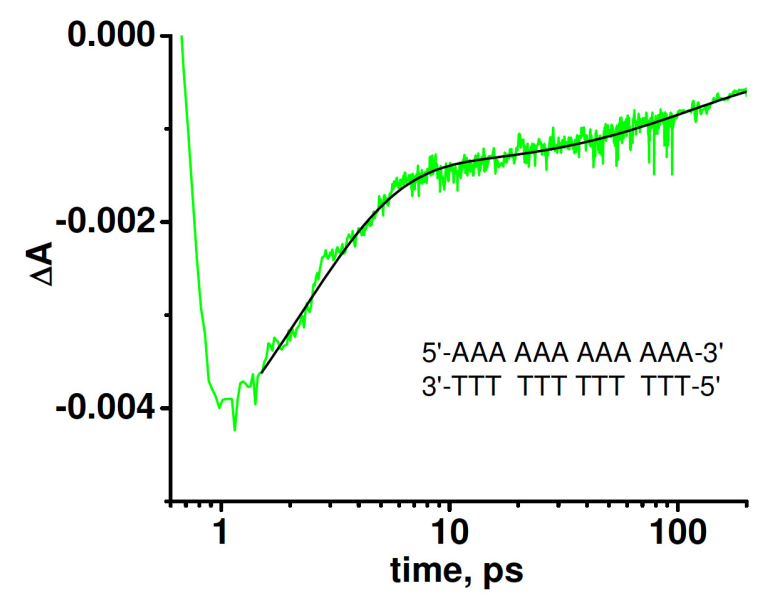

(b)

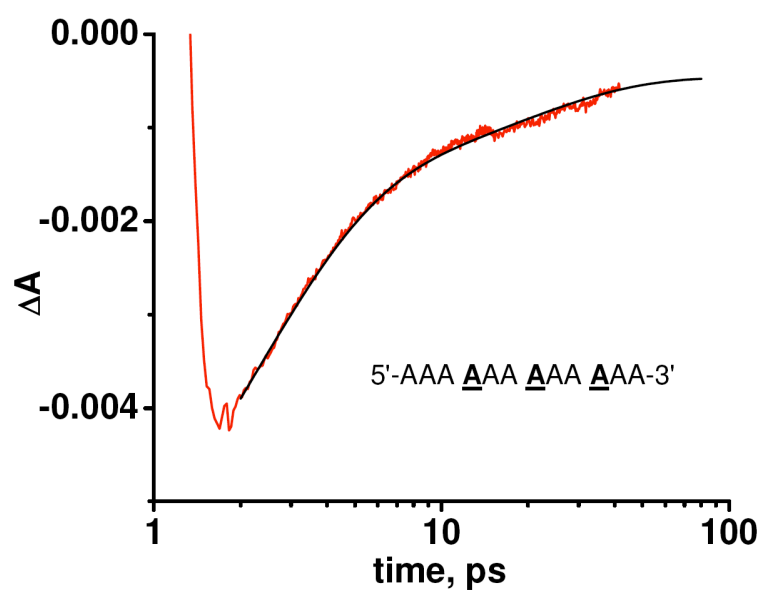

(d)

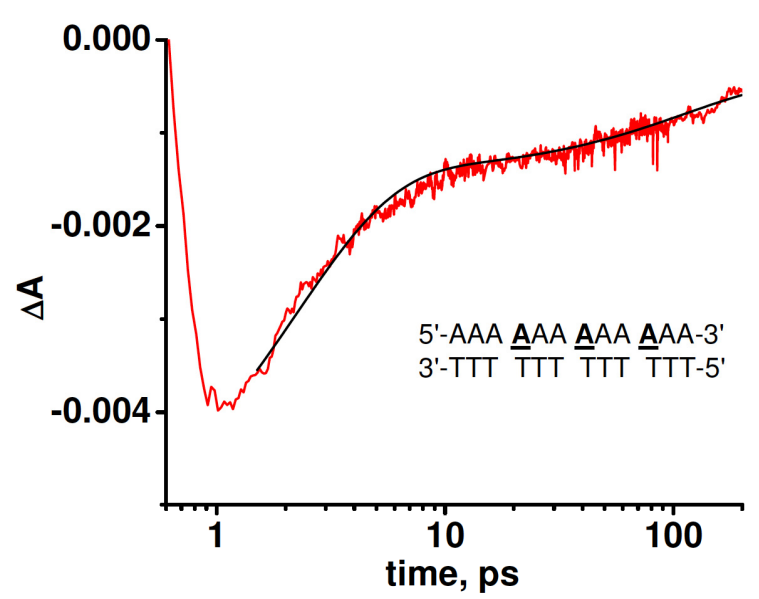

Fig. (5). Transient absorption data (pump $260 \mathrm{~nm}$, probe $260 \mathrm{~nm}$ ) for adenines in single-stranded (a) DNA and (b) LNA and hybridized with their respective complements (c and $\mathbf{d}$, respectively). The traces of the single-stranded samples confirm the dependence of excited state decay on conformation while those of the hybridized samples show the masking thymine repopulation of the electronic ground state occurring via an unidentified singlet intermediate [11] not evident with the $440 \mathrm{~nm}$ probe (Fig. 4c).

cited electronic states $\left(\tau_{1}, \tau_{2}\right)$ occurring faster in LNA than in DNA, because the less restrictive configuration of stacked bases in the former facilitates CIs with the electronic ground state via non-planar base geometries as discussed above, excited electronic states that form are also constrained to be more localized due to the unfavorable base stacking geometry of LNA for excited state delocalization even though they may be energetically somewhat favored due to reduced electron correlation as discussed earlier.

Long-lived excited states are often considered to elevate the risk of photoproduct formation because chemical bonds can be formed before excess energy is funneled to the ground state $[20,30,37]$. In the present context, fewer delocalized excitons and faster excited state relaxation in LNA suggests a lower probability of adenine in poly(dA) sequences relaxing from an exciton to a stable state different from the ground state $S_{0}$ and hence a lower probability of $\mathrm{UV}$-induced photodamage in LNA compared to DNA. This is consistent with findings that RNA, which adopts the Aform conformation, is more resistant to UV damage than
DNA [65]. However, the view that long-lived excited states are deleterious contrasts with the ultrafast and high yield photodimerization of thymine [66], high yield formation of long-lived excited states in adenine strands [11], and the low yield of adenine photoproducts [67]. The ultrafast photodimerization of thymine, especially in single strands, is attributed to the presence of pairs of adjacent thymines already properly oriented for dimerization [66]. Since ultrafast nonradiative relaxation processes from localized excited states is the major pathway of energy dissipation in excited nucleic acid bases $[23,29,30]$, it raises the possibility that long-lived delocalized excited states may in fact provide a mechanism for sequestering excess energy until it can be dissipated safely. For example, strand localization of excitation has been reported based on the similarities between ESA intensities and decay profiles in poly $(\mathrm{dA}) \cdot \operatorname{poly}(\mathrm{dT})$ and poly(dA), poly(dT) being different [11]; theoretical calculations with a lattice fermion model incorporating parallel (intrachain), perpendicular (cross-chain), and diagonal crosschain terms show delocalized excitons to have weak mixing 
between the poly(dA) and poly(dT) strands [68]; and theoretical modeling shows, over the $\sim 300$ fs time period examined, coherent exciton migration on the adenine strand of poly(dA)'poly(dT) [22] while excitation on the thymine strand devolves into charges that migrate along that strand $[22,68]$. Delocalization may also serve to prevent trapping of energy with subsequent increased probability of photoreaction [55]. Excess energy dissipation can thus occur by migration of the delocalized excitation in adenine stacks with B-form stacking geometry to poorly-stacked or end regions where non-planar base geometries can facilitate ultrafast relaxation. Thus long-lived excited states appear to be "protective", at least in adenine stacks, and the character and dynamics of these long-lived excited states, given the local geometry, may therefore be crucial in explaining their protective effects. The lower susceptibility of RNA to photodamage may therefore reflect the fact that bases in A-from are not positioned favorably for photoadduct formation.

\section{CONCLUSIONS}

The first experimental investigation of the excited state dynamics in LNA, along with their DNA analogs, permitted a comparison of these dynamics in single-stranded and double-stranded oligomers of identical primary structures, but different secondary structures. A high degree of geometric overlap between stacked adjacent adenines favors delocalized excited state formation both in the Franck-Condon region and in the course of subsequent evolution. In contrast, a lack of geometric overlap permits ultrafast decay of excited states to the electronic ground state. Therefore adenine bases stacked in a manner that evinces a high degree of geometric overlap ( $\mathrm{B}^{*}$-form) are more readily cooperatively excited, these excited states are more likely to remain delocalized, and because stacking structurally constrains non-planar base geometries, they cannot decay easily to the electronic ground state, thus they have relatively long lifetimes compared to those stacked with a lesser degree of geometric overlap (Aform). Our results show clearly that base stacking geometry determines excited state dynamics in nucleic acids. Thus they confirm and extend observations from poly $(\mathrm{rC})$ where duplex and quadruplex geometries were induced with $\mathrm{pH}$ and long excited state lifetimes were found to occur in the hemiprotonated, self-associated, duplex structure - the structure with more base overlap [69]. The data also provide evidence of delocalization over at least two, but potentially more, adenine bases and that delocalization may be more extensive during the early part of the excited stage.

The degree of geometric overlap and the relative orientation of neighboring bases are important parameters affecting exciton formation and dissipation and hence affect the likelihood of photoproduct formation. A better understanding of the precise effects of these parameters on excited state dynamics will therefore contribute to our understanding of the roles that secondary structures play in the susceptibility of genetic material to the formation of photo-induced lesions.

\section{ACKNOWLEDGEMENTS}

We thank Curtis Hughesman and Yee Chee Lim for help preparing the samples. Instrumentation and infrastructure were provided by the UBC Laboratory for Advanced Spectroscopy and Imaging Research (LASIR) and Laboratory for Molecular Biophysics (LMB). Funding was provided by the
Natural Sciences and Engineering Research Council (NSERC), the Canadian Institutes of Health Research (CIHR), the Canada Foundation for Innovation (CFI) and the British Columbia Knowledge Development Fund (BCKDF).

\section{REFERENCES}

[1] Rich A, Kasha M. The $\mathrm{n} \rightarrow \pi^{*}$ transition in nucleic acids and polynucleotides. J Am Chem Soc 1960; 82: 6197-9.

[2] Hug W, Tinoco I. Electronic spectra of nucleic acid bases. I. Interpretation of in-plane spectra with the aid of all valence electron MO-CI calculations. J Am Chem Soc 1973; 95: 2803-13.

[3] Ingwall JS. Circular dichroism of nucleosides. I. Anomeric pairs of the D-pentofuranosides of adenine. J Am Chem Soc 1972; 94: 5487-95.

[4] Miles DW, Robins MJ, Robins RK, Eyring H. Circular dichroism of nucleoside derivatives, VI. The optically active bands of adenine nucleoside derivatives. Proc Natl Acad Sci USA 1969; 62: 22-9.

[5] Holmen A, Broo A, Albinsson B, Norden B. Assignment of electronic transition moment directions of adenine from linear dichroism measurements. J Am Chem Soc 1997; 119: 12240-50.

[6] Marian CM. A new pathway for the rapid decay of electronically excited adenine. J Chem Phys 2005; 122: 104314.

[7] Ritze H-H, Lippert H, Samoylova E, et al. Relevance of $\pi \sigma^{*}$ states in the photoinduced processes of adenine, adenine dimer, and adenine-water complexes. J Chem Phys 2005; 122: 2243201-9.

[8] Samoylova E, Lippert H, Ullrich S, Hertel IV, Radloff W, Schultz $\mathrm{T}$. Dynamics of photoinduced processes in adenine and thymine base pairs. J Am Chem Soc 2005; 127: 1782-6.

[9] Serrano-Andres L, Merchan M, Borin AC. Adenine and 2aminopurine: paradigms of modern theoretical photochemistry. Proc Natl Acad Sci USA 2006; 103: 8691-6.

[10] Sinha RP, Häder D-P. UV-induced DNA damage and repair: a review. Photochem Photobiol Sci 2002; 1: 225-36.

[11] Crespo-Hernández CE, Cohen B, Kohler B. Base stacking controls excited state dynamics in A.T DNA. Nature 2005; 436: 1141-4.

[12] Malone RJ, Miller AM, Kohler B. Singlet excited-state lifetimes of cytosine derivatives measured by femtosecond transient absorption. Photochem Photobiol 2003; 77: 158-64.

[13] Wan C, Fiebig T, Schiemann O, Barton JK, Zewail AH. Femtosecond direct observation of charge transfer between bases in DNA. Proc Natl Acad Sci USA 2000; 97: 14052-5.

[14] Xu D-G, Nordlund TM. Sequence dependence of energy transfer in DNA oligonucleotides. Biophys J 2000; 78: 1042-58.

[15] Cantor CR, Schimmel PR. The conformation of biological macromolecules. WH Freeman: San Francisco; 1980.

[16] Saenger W. Principles of nucleic acid structure. Springer: New York; 1984.

[17] Parthasarathi R, Subramanian V. Stacking interactions in benzene and cytosine dimers: from molecular electron density perspective. Struct Chem 2005; 16: 243-55.

[18] Sponer J, Berger I, Spackova N, Leszczynski J, Hobza P. Aromatic base stacking in DNA: from ab initio calculations to molecular dynamics simulations. J Biomol Struct Dyn 2000; Conversation 11: 383-407.

[19] Sponer J, Leszczynski J, Hobza P. Hydrogen bonding, stacking and cation binding of DNA bases. J Mol Struct 2001; 573: 43-53.

[20] Hu L-H, Zhao Y, Wang F, et al. Are adenine strands helical Haggregates? J Phys Chem B 2007; 111: 11812-6.

[21] Lu X-J, Olson WK. 3DNA: a software package for the analysis, rebuidling and visualization of three-dimensional nucleic acid structures. Nucleic Acids Res 2003; 31: 5108-21.

[22] Bittner ER. Frenkel exciton model of ultrafast excited state dynamics in AT DNA double helices. J Photoch Photobio A 2007; 190: 328-34.

[23] Shukla MK, Leszczynski J. Electronic spectra, excited state structures and interactions of nucleic acid bases and base assemblies: a review. J Biomol Struct Dyn 2007; 25: 93-118.

[24] Shukla MK, Kuramshina GM, Leszczynski J. Evidence of structural non-planarity in excited state: new findings provided by vibrational analysis of the guanine-cytosine base pair. Chem Phys Lett 2007; 447: 330-4.

[25] Shukla MK, Leszczynski J. A theoretical investigation of excitedstate properties of the adenine-uracil base pair. J Phys Chem 2002; 106: 1011-8. 
[26] Schwalb NK, Temps F. Ultrafast electronic relaxation in guanosine is promoted by hydrogen bonding with cytidine. J Am Chem Soc 2007; 129: 9272-3.

[27] Sobolewski AL, Domcke W. Ab initio studies on the photophysics of the guanine-cytosine base pair. Phys Chem Chem Phys 2004; 6: 2763-71

[28] Abo-Riziq A, Grace L, Nir E, Kabelac M, Hobza P, de Vries MS. Photochemical selectivity in guanine-cytosine base-pair structures. Proc Natl Acad Sci USA 2005; 102: 20-3.

[29] Pecourt J-ML, Peon J, Kohler B. Ultrafast internal conversion of electronically excited RNA and DNA nucleosides in water. J Am Chem Soc 2000; 122: 9348-9.

[30] Pecourt J-ML, Peon J, Kohler B. DNA excited-state dynamics: ultrafast internal conversion and vibrational cooling in a series of nucleosides. J Am Chem Soc 2001; 123: 10370-8.

[31] Brovchenko I, Krukau A, Oleinikova A. Water percolation governs polymorphic transitions and conductivity of DNA. Phys Rev Lett 2006; 97: 137801

[32] Vorlickova M. Conformational transitions of alternating purinepyrimidine DNAs in perchlorate ethanol solutions. Biophys J 1995; 69: $2033-43$

[33] Starikov EB, Lewis JP, Sankey OF. Base sequence effects on charge carrier generation in DNA: a theoretical study. Int J Mod Phys B 2005; 19: 4331-57.

[34] Nordlund TM. Sequence, structure and energy transfer in DNA. Photochem Photobiol 2007; 83: 625-36

[35] Emanuele E, Markovitsi D, Millie P, Zakrzewksa K. UV spectra and excitation delocalization in DNA: influence of the spectral width. ChemPhysChem 2005; 6: 1387-92.

[36] Santoro F, Barone V, Improta R. Influence of base stacking on excited-state behavior of polyadenine in water, based on timedependent density functional calculations. Proc Natl Acad Sci USA 2007; 104: 9931-6.

[37] Buchvarov I, Wang Q, Raytchev M, Trifonov A, Fiebig T. Electronic energy delocalization and dissipation in single- and doublestranded DNA. Proc Natl Acad Sci USA 2007; 104: 4794-7.

[38] Markovitsi D, Talbot F, Gustavsson T, Onidas D, Lazzarotto E, Marguet S. Complexity of excited-state dynamics in DNA. Nature 2006; 441: E7-E8.

[39] Markovitsi D, Onidas D, Talbot F, Marguet S, Gustavsson T, Lazzarotto E. UVB/UVC induced processes in model DNA helices studied by time-resolved spectroscopy: pitfalls and tricks. J Photoch Photobio A 2006; 183: 1-8.

[40] Markovitsi D, Gustavsson T, Talbot F. Excited states and energy transfer among DNA bases in double helices. Photochem Photobiol Sci 2007; 6: 717-24

[41] Lewis FD, Liu X, Wu Y, Zuo X. Stepwise Evolution of the Structure and Electronic Properties of DNA. J Am Chem Soc 2003; 125: 12729-31

[42] Nix MGD, Devine AL, Cronin B, Ashfold MNR. Ultraviolet photolysis of adenine: dissociation via the ${ }^{1} \pi \sigma^{*}$ state. J Chem Phys 2007; 126: 124312.

[43] Satzger H, Townsend D, Zgierski MZ, Patchkovskii S, Ullrich S, Stolow A. Primary processes underlying the photostability of isolated DNA bases: adenine. Proc Natl Acad Sci USA 2006; 103: 10196-201.

[44] Kwok W-M, Ma C, Phillips DL. Femtosecond time- and wavelength-resolved fluorescence and absorption spectroscopic study of the excited states of adenosine and an adenine oligomer. J Am Chem Soc 2006; 128: 11894-905.

[45] Starikov EB. Importance of charge transfer excitations in DNA in electron spectrum: a ZINDO semiempirical qunatum-chemical study. Mod Phys Lett B 2004; 18: 825-31.

[46] Alexeev DG, Lipanov AA, Skuratovskii IY. Poly(dA)·poly(dT) is a B-type double helix with a distinctively narrow minor groove. Nature 1987; 325: 821-3

[47] Aymami J, Coll M, Frederick CA, Wang AH-J, Rich A. The propeller DNA conformation of poly(dA).poly(dT). Nucleic Acids Res 1989; 17: 3229-45.
[48] Nelson HCM, Finch JT, Luisi BF, Klug A. The structure of an oligo(dA). oligo(dT) tract and its biological implications. Nature 1987; 330: 221-6.

[49] Crespo-Hernández C, Kohler B. Influence of secondary structure on electronic energy relaxation in adenine homopolymers. J Phys Chem B 2004; 108: 11182-8

[50] Koshkin AA, Singh SK, Nielsen P, et al. LNA (locked nucleic acids): synthesis of the adenine, cytosine, guanine, 5methylcytosine, thymine and uracil bicyclonucleoside monomers, oligomerisation, and unprecedented nucleic acid recognition. Tetrahedron 1998; 54: 3607-30.

[51] Nielsen KE, Rasmussen J, Kumar R, Wengel J, Jacobsen JP, Petersen M. NMR studies of fully modified locked nucleic acid (LNA) hybrids: solution structure of an LNA:RNA hybrid and characterizartion of LNA:DNA hybrid. Bioconjug Chem 2004; 15: 449-57.

[52] Premilat S, Albiser G. X-Ray fibre diffraction study of an elevated temperature structure of poly(dA)poly(dT). J Mol Biol 1997; 274 64-71.

[53] Colon L, Crespo-Hernández CE, Oyola R, Garcia C, Arce R. Role of sequence and conformation on the photochemistry and the photophysics of A-T DNA dimers: an experimental and computational approach. J Phys Chem B 2006; 110: 15589-96.

[54] Dewey TG, Turner DH. Laser temperature-jump study of stacking in adenylic acid polymers. Biochemistry 1979; 18: 5757-62

[55] Ritze H-H, Hobza P, Nachtigallova D. Electronic coupling in the excited electronic state of stacked DNA base homodimers. Phys Chem Chem Phys 2007; 9: 1672-5.

[56] Broo A. A theoretical investigation of the physical reason for the very different luminescence properties of the two isomers adenine and 2-aminopurine. J Phys Chem A 1998; 102: 526-31.

[57] Bouvier B, Dognon J-P, Lavery R, et al. Influence of conformational dynamics on the exciton states of DNA oligomers. J Phys Chem B 2003; 107: 13512-22.

[58] Markovitsi D, Onidas D, Gustavsson T, Talbot F, Lazarotto E. Collective behavior of Franck-Condon excited states and energy transfer in DNA double helices. J Am Chem Soc 2005; 127: 171301.

[59] Hagerman KR, Hagerman PJ. Helix rigidity of DNA: the meroduplex as an experimental paradigm. J Mol Biol 1996; 260: 207-23.

[60] Mills JB, Hagerman PJ. Origin of the intrinsic rigidity of DNA. Nucleic Acids Res 2004; 32: 4055-9.

[61] Mills JB, Vacano E, Hagerman PJ. Flexibility of single-stranded DNA: use of gapped duplex helices to determine the persistence lengths of poly(dT) and poly(dA). J Mol Biol 1999; 285: 245-57.

[62] Pande V, Nilsson L. Insights into structure, dynamics and hydration of locked nucleic acid (LNA) strand-based duplexes from molecular dynamics simulations. Nucleic Acids Res 2008; 36: 1508-16.

[63] Bonvin AMJJ, Sunnerhagen M, Otting G, van Gunsteren WF Water molecules in DNA recognition II: a molecular dynamics view of the structure and hydration of the trp operator. J Mol Biol 1998; 282: 859-73

[64] Eisinger J, Shulman RG. Excited electronic states of DNA. Science 1968; 161: 1311-9.

[65] Kundu LM, Linne U, Marahiel M, Carell T. RNA is more UV resistant than DNA: the formation of UV-induced DNA lesions is strongly sequence and conformation dependent. Chem-Eur J 2004; 10: 5697-705.

[66] Schreier WJ, Schrader TE, Koller FO, et al. Thymine dimerization in DNA is an ultrafast photoreaction. Science 2007; 315: 625-9.

[67] Pattison DI, Davies MJ. Actions of ultraviolet light on cellular structures. In: Bignold LP, Ed., Cancer: cell structures, carcinogens and genomic instability, Birkhauser Verlag, Basel, 2006; pp. 13157.

[68] Bittner ER. Lattice theory of ultrafast excitonic and charge-transfer dynamics in DNA. J Chem Phys 2006; 125: 094909.

[69] Cohen B, Larson MH, Kohler B. Ultrafast excited-state dynamics of RNA and DNA C tracts. Chem Phys 2008; 350: 165-74. 